arXiv:1763650 [gr-qc] 08.02.2017 (v1.00)

\title{
Four-form field versus fundamental scalar field
}

\author{
M.K. Savelainen* \\ Low Temperature Laboratory, Department of Applied Physics, Aalto University, \\ PO Box 15100, FI-00076 Aalto, Finland
}

\begin{abstract}
A modified-gravity theory with a four-form field strength $F$, a variable gravitational coupling parameter $G(F)$, and a standard matter action is considered here. Maxwell and Einstein equations are now derived when including to action also derivates of $F$. The energy momentum tensor of the 4-form field contains both the part, which is typical for the fundamental (pseudo)scalar, and the part, which cancels the divergent contribution of the zero-point energies of quantum fields to the vacuum energy and thus leads to the natural nullification of the cosmological constant in Minkowski vacuum.

PACS numbers: 04.20.Cv, 98.80.Jk, 95.35.+d, 95.36.+x
\end{abstract}

Keywords: general relativity, cosmology, dark matter, dark energy

*Electronic address: matti.savelainen@aalto.fi 


\section{INTRODUCTION}

The physics of the many-body condensed systems provides numerous hints for high energy physics and cosmology. For example, the Gor'kov theory of superconductivity [1] opened the route to the construction of the relativistic quantum field models, [2 [4] in which the Higgs bosons are composite objects being analogs of the amplitude modes in superconductors. [5] The connection between the topologically protected Weyl fermions in topological materials - superfluids, semimetals and superconductors of the Weyl type - and chiral particles in Standard Model of particle physics also suggests that the Standard Model is an effective theory, where the Weyl fermions, gauge fields and gravity emerge in the vicinity of the topologically protected Weyl points in the spectrum of the quantum vacuum. [6 8 ]

Another connection between the ground state of the many-body condensed matter system and the quantum vacuum is revealed when one considers the energy of the quantum vacuum, which contributes to the cosmological constant. The discrepancy between the observed almost zero value of the vacuum energy and its estimation in terms of the zero point energy of fermionic and bosonic quantum fields provides the cosmological constant problem. Most plausibly the huge discrepancy of about 120 orders is the result of the estimations, which have been based on low-energy effective field theory. While the condensed matter teaches us that such quantity, as the ground state energy of the quantum systems, can be computed only within a full microscopic quantum theory. Within such theories the condensed matter systems demonstrate, that if the given system is close to equilibrium, the properly determined thermodynamic energy is close to zero. In a full equilibrium the huge contribution of the zero point motion is completely cancelled by the microscopic (correspondingly trans-Planckian) degrees of freedom.

Unfortunately, to date, we do not have any microscopic theory of the quantum vacuum. However, again the condensed matter demonstrates to us that the microscopic degrees of freedom can be also described in terms of their own effective macroscopic variables, which do not depend mush on the detailed microscopic structure of the system, such as the density of atoms in the many-body quantum systems. The Lorentz invariant analog of such macroscopic approach is represented by the so-called $q$-theory. [9 11] It also provides the general description of the quantum vacuum, and its equations do not depend on the choice of the vacuum variables, and on the microscopic (trans-Planckian) details. As in condensed matter, the sub-Planckian and trans-Planckian contributions to the energy of the fully equilibrium vacuum are naturally canceled by the thermodynamic argument without fine-tuning, in spite of the huge contribution of the zero point energies.

The particular useful choice for the vacuum variable is the 4-form field, [12 17] which satisfies all the requirements needed for the description of the quantum vacuum, especially if instead of the quadratic form in the $F$ field, one use the general function of $F$. Later it 
became clear that the $q$-theory must be extended to include the derivatives of the $q$-field. In particular this is important for the consideration of the possible origin of dark matter, and for the consideration of the inhomogeneous states of the vacuum, such as interfaces between the vacua. The latter is important if the Universe is at the coexistence point, where different vacua have the same energy. This is in the origin of the so-called multiple point principle. 18 22].

This extension was done in Refs. [23, 24], where the derivatives of the $q$-field has been added to the action, and it was shown that as a result the oscillations of the $q$-field during cosmological evolution produce the kind of dark matter. In this current paper we, in more detail, derive the Einstein equations that are presented as equations (5) and (6) in the article [23]. Also derivation of equation (4) is included. The gravity field is presented here in a more general way like in [10].

\section{THE ACTION FOR GRAVITY WITH $F$ FIELD WITH GRADIENTS AND VARIABLE GRAVITATIONAL COUPLING}

The action for the 4-form field interacting with the gravitational field has the following form $(\hbar=c=1)$ :

$$
\begin{gathered}
S=-\int_{\mathbb{R}^{4}} d^{4} x \sqrt{|g|}\left(\frac{R}{16 \pi G(F)}+\epsilon(F)+\frac{1}{8} K(F) \nabla^{\alpha} F^{2} \nabla_{\alpha} F^{2}+\mathcal{L}^{\mathrm{SM}}\right), \\
F_{\kappa \lambda \mu \nu} \equiv \nabla_{[\kappa} A_{\lambda \mu \nu]}, \quad F^{2} \equiv-\frac{1}{4 !} F_{\kappa \lambda \mu \nu} F^{\kappa \lambda \mu \nu} \\
F_{\kappa \lambda \mu \nu}=F \sqrt{|g|} e_{\kappa \lambda \mu \nu}, \quad F^{\kappa \lambda \mu \nu}=F e^{\kappa \lambda \mu \nu} / \sqrt{|g|}
\end{gathered}
$$

$\nabla_{\mu}$ denotes a covariant derivative and a square bracket around spacetime indices complete anti-symmetrization. $\nabla^{\alpha} F^{2} \nabla_{\alpha} F^{2}$ is $g^{\alpha \beta} \nabla_{\beta} F^{2} \nabla_{\alpha} F^{2} . K(F)$ is some factor depending on $F$

only (here not on its derivatives). $\mathcal{L}^{\mathrm{SM}}$ is the Lagrange density of the fields of the standard model (SM) of elementary particle physics. Throughout, we use natural units with $c=\hbar=1$ and take the metric signature $(-+++)$.

Variation over $A_{\lambda \mu \nu}$ gives the Maxwell equations, see Appendix:

$$
\nabla_{\kappa}\left(\frac{R}{16 \pi} \frac{d G^{-1}(F)}{d F}+\frac{d \epsilon(F)}{d F}+\frac{1}{8} \frac{d K(F)}{d F} \partial^{\alpha} F^{2} \partial_{\alpha} F^{2}-\frac{1}{2} F \nabla^{\alpha}\left(K(F) \partial_{\alpha} F^{2}\right)\right)=0 .
$$

From Maxwell equation (2.2) we get

$$
\frac{R}{16 \pi} \frac{d G^{-1}(F)}{d F}+\frac{d \epsilon(F)}{d F}+\frac{1}{8} \frac{d K(F)}{d F} \partial^{\alpha} F^{2} \partial_{\alpha} F^{2}-\frac{1}{2} F \nabla^{\alpha}\left(K(F) \partial_{\alpha} F^{2}\right)=\mu,
$$

where $\mu$ is the integration constant. It plays the role of chemical potential, which is thermodynamically conjugate to $F$. It is convenient to set

$$
C(F)=F^{2} K(F),
$$


which gives for Maxwell equations:

$$
\frac{R}{16 \pi} \frac{d G^{-1}(F)}{d F}+\frac{d \epsilon(F)}{d F}-\frac{1}{2} \frac{d C(F)}{d F} \partial^{\alpha} F \partial_{\alpha} F-C(F) \square F=\mu .
$$

Variation over the metric $g^{\mu \nu}$ gives the generalized Einstein equations, see Appendix:

$$
\begin{aligned}
& \frac{1}{8 \pi G(F)}\left(R_{\mu \nu}-\frac{1}{2} R g_{\mu \nu}\right)+\frac{1}{16 \pi} F \frac{d G^{-1}}{d F} R g_{\mu \nu}+ \\
& +\frac{1}{8 \pi}\left(\nabla_{\mu} \nabla_{\nu} G^{-1}(F)-g_{\mu \nu} \square G^{-1}(F)\right)-\left(\epsilon(F)-F \frac{d \epsilon(F)}{d F}\right) g_{\mu \nu}+ \\
& -\frac{1}{2} C(F) g_{\mu \nu} \nabla_{\alpha} F \nabla^{\alpha} F-\frac{1}{2} F \frac{d C(F)}{d F} \nabla_{\alpha} F \nabla^{\alpha} F g_{\mu \nu}+ \\
& +C(F) \nabla_{\mu} F \nabla_{\nu} F-C(F) \square F g_{\mu \nu}+T_{\mu \nu}^{(\mathrm{SM})}=0
\end{aligned}
$$

which can be simplified using Eq.(2.5):

$$
\begin{aligned}
& \frac{1}{8 \pi G(F)}\left(R_{\mu \nu}-\frac{1}{2} R g_{\mu \nu}\right)+\frac{1}{8 \pi}\left(\nabla_{\mu} \nabla_{\nu} G^{-1}(F)-g_{\mu \nu} \square G^{-1}(F)\right)+ \\
& -(\epsilon(F)-\mu F) g_{\mu \nu}-\frac{1}{2} C(F) g_{\mu \nu} \nabla_{\alpha} F \nabla^{\alpha} F+C(F) \nabla_{\mu} F \nabla_{\nu} F+T_{\mu \nu}^{(\mathrm{SM})}=0 .
\end{aligned}
$$

For the constant gravitational coupling $G(F)$ these equations are reduced to the corresponding equation in the article [23],

The Einstein equation (2.7) shows that the contribution of the 4-form field to the gravitating energy-momentum tensor is given by

$$
T_{\alpha \beta}^{(F)}=\left(C(F) \nabla_{\alpha} F \nabla_{\beta} F-\frac{1}{2} g_{\alpha \beta} C(F) \nabla_{\mu} F \nabla^{\mu} F\right)-g_{\alpha \beta}(\epsilon(F)-\mu F) .
$$

The first term on the RHS of Eq.(2.8) corresponds to the energy-momentum tensor of the conventional scalar field. However, the second term on the RHS of Eq.(2.8) demonstrates the consequence of the fact that $F$ is not a fundamental (pseudo)scalar but a composite object made of the gauge field $A_{\kappa \lambda \mu}$ and the metric $g_{\mu \nu}$.

\section{CONCLUSION}

There are two faces of the 4-form field, as follows from Eq.(2.8): it has the signature of the (pseudo)scalar and the signature of the conserved quantity, which characterizes the deep quantum vacuum. Due to the latter, the contribution to the vacuum energy from the $F$-field is $\epsilon(F)-\mu F$, instead of the conventional term $\epsilon(F)$ in the theory of the fundamental

scalar field. This difference allows us to avoid the fine-tuning problem [25] in estimation of the contribution of the vacuum energy to the cosmological constant in Minkowski vacuum. 
While in the scalar field approach the nullification of the diverging contribution of zero-pointenergies to the vacuum energy density $\epsilon(F)$ looks artificial, in the $F$-theory the term $\epsilon(F)$ is automatically cancelled by the counter term $-\mu F$. This cancellation is required by the Gibbs-Duhem identity, which is applicable to any equilibrium ground state, including the one of the physical vacuum. As a result the diverging contribution of zero-point-energies of quantum fields is fully compensated by microscopic degrees of freedom, which are effectively described by the vacuum field $F$. The proper vacuum energy density entering the Einstein gravitational equation (2.7) as cosmological constant is zero in a full equilibrium at zero temperature, $\Lambda=\epsilon(F)-\mu F=0$.

The same nullification happens if in addition to the $F$-field there is the vacuum contribution of the matter fields [9]. For example, if there is the fundamental scalar field $\Phi$ in the matter sector with the vacuum energy density $\epsilon_{\text {matter }}(\Phi)$, the total vacuum energy in equilibrium will be also cancelled, $\Lambda=\epsilon(F)+\epsilon_{\text {matter }}(\Phi)-\mu F=0$. The compensation of the energy density comes again from the microscopic degrees of freedom. The chemical potential $\mu$ of the vacuum field is self-tuned to the matter fields.

It is important that the form of the Einstein equation in terms of the $F$-field (2.7) and the form of the equation (2.5) for the $F$-field are general, and do not depend much on the origin of the vacuum field. The only specific property of the $F$-field, which leads to the counter term $-\mu F$ in the cosmological constant, is the appearance of chemical potential $\mu$ for $F$-field in (2.5). The existence of the chemical potential in the system is the typical consequence of the conservation law. Thus the form of the equations is the consequence of the conservation law, and does not depend on the particular choice of the vacuum field. The $F$-field is the particular representation of the vacuum field, which obey the proper conservation law.

The obtained equations are applicable for different problems such as: (i) relaxation of the vacuum energy in the expanding Universe; (ii) the internal structure of the black hole including the structure of the singularity; (iii) investigation of topological and non topological objects; etc.

\section{ACKNOWLEDGMENTS}

I would like to express my deep gratitude to Professor G.V. Volovik for his guidance. This work has been supported by the Academy of Finland (Project No. 284594). 


\section{Appendix}

We divide action into three parts:

$$
\begin{aligned}
S & =S_{0}+S_{1}+S_{2}, \\
S_{0} & =-\int_{\mathbb{R}^{4}} d^{4} x \sqrt{|g|}\left(\frac{R}{16 \pi G(F)}+\epsilon(F)\right), \\
S_{1} & =-\int_{\mathbb{R}^{4}} d^{4} x \sqrt{|g|}\left(\frac{1}{8} K(F) \nabla^{\alpha} F^{2} \nabla_{\alpha} F^{2}\right), \\
S_{2} & =-\int_{\mathbb{R}^{4}} d^{4} x \sqrt{|g|} \mathcal{L}^{\mathrm{SM}} .
\end{aligned}
$$

Next we variate $S_{1}$ with respect to the three-form gauge field $A$. Lets first open $S_{1}$.

$$
\begin{aligned}
S_{1}= & -\int_{\mathbb{R}^{4}} d^{4} x \sqrt{|g|} \frac{1}{8} K(F) g^{\alpha \beta} \times \\
& \times \nabla_{\beta}\left(\nabla_{[\kappa} A_{\lambda \mu \nu]} \nabla^{[\kappa} A^{\lambda \mu \nu]}\right) \nabla_{\alpha}\left(\nabla_{[\kappa} A_{\lambda \mu \nu]} \nabla^{[\kappa} A^{\lambda \mu \nu]}\right) \frac{1}{4 !^{2}} .
\end{aligned}
$$

$\underline{\lambda}, \underline{\mu}$ and $\underline{\nu}$ are from now on specific index values and usual sum convention not used for them. This underlined set of indices defines the special direction, to which we make the variation $\delta A \underline{\lambda \mu \nu}$. Note that now all other $\delta A^{\lambda \mu \nu}$ and $\nabla \delta A^{\lambda \mu \nu}$ are zero if $\lambda \neq \underline{\lambda}$ or $\mu \neq \underline{\mu}$ or $\nu \neq \underline{\nu}$. This convention is taken just to make summing in the following derivation easier.

$$
\begin{aligned}
& S_{1}+\delta S_{1}=-\int_{\mathbb{R}^{4}} d^{4} x \sqrt{|g|} \frac{1}{8} K(F+\delta F) \cdot \frac{1}{4 !^{2}} \times \\
& \left.\times g^{\alpha \beta} \nabla_{\beta}\left(\nabla_{[\kappa} A_{\lambda \mu \nu}+\delta A_{\underline{\lambda \mu \nu}]} \nabla^{[\kappa} A^{\lambda \mu \nu}+\delta A^{\lambda \mu \nu}\right]\right) \times \\
& \left.\times \nabla_{\alpha}\left(\nabla_{[\hat{\kappa}} A_{\dot{\lambda} \dot{\mu} \dot{\nu}}+\delta A_{\underline{\lambda \mu \nu}]} \nabla^{[\hat{\kappa}} A^{\dot{\lambda} \mu \dot{\mu}}+\delta A^{\lambda \mu \nu}\right]\right) \\
& =-\int_{\mathbb{R}^{4}} d^{4} x \sqrt{|g|} \frac{1}{8} K(F+\delta F) \cdot \frac{1}{4 !^{2}} \times \\
& \left.\times g^{\alpha \beta} \nabla_{\beta}\left[\nabla_{[\kappa} A_{\lambda \mu \nu]} \nabla^{[\kappa} A^{\lambda \mu \nu]}+\nabla_{[\kappa} A_{\lambda \mu \nu]} \nabla^{[\kappa} \delta A^{\lambda \mu \nu}\right]+\nabla_{[\kappa} \delta A_{\underline{\lambda \mu \nu}]} \nabla^{[\kappa} A^{\lambda \mu \nu]}+2 \text { nd ord }\right] \times
\end{aligned}
$$

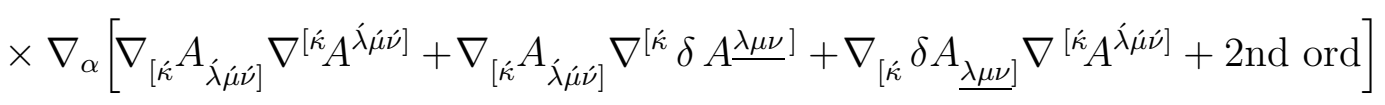




$$
\begin{aligned}
& S_{1}+\delta S_{1} \approx-\int_{\mathbb{R}^{4}} d^{4} x \sqrt{|g|} \frac{1}{8}\left(K(F)+\frac{d K(F)}{d F} \delta F\right) \times \\
& \times g^{\alpha \beta}\left[\nabla_{\beta} F^{2}+2 \nabla_{\beta}\left(\frac{F_{\kappa \lambda \mu \nu} \nabla^{\kappa} \delta A \underline{\lambda \mu \nu}}{-4 !} \cdot 24\right)\right]\left[\nabla_{\alpha} F^{2}+2 \nabla_{\alpha}\left(\frac{F_{\kappa \dot{\alpha} \mu \mu \nu} \nabla^{\kappa} \delta A \underline{\lambda \mu \nu}}{-4 !} \cdot 24\right)\right] \\
& =S_{1}-\int_{\mathbb{R}^{4}} d^{4} x \sqrt{|g|} \frac{1}{8} \frac{d K(F)}{d F} \delta F \nabla^{\alpha} F^{2} \nabla_{\alpha} F^{2}+ \\
& -\int_{\mathbb{R}^{4}} d^{4} x \sqrt{|g|} \frac{1}{8} K(F) g^{\alpha \beta}\left[2 \nabla_{\beta}\left(-F_{\kappa \lambda \mu \nu} \nabla^{\kappa} \delta A \frac{\lambda \mu \nu}{)} \nabla_{\alpha} F^{2}\right]+\right. \\
& -\int_{\mathbb{R}^{4}} d^{4} x \sqrt{|g|} \frac{1}{8} K(F) g^{\alpha \beta}\left[2 \nabla_{\alpha}\left(-F_{\kappa \dot{\alpha} \dot{\mu} \dot{\nu}} \nabla^{\kappa} \delta A^{\lambda \mu \nu}\right) \nabla_{\beta} F^{2}\right]+2 \text { nd ord . }
\end{aligned}
$$

For $\delta F$ we get

$$
\delta F=-\frac{1}{F} F_{\kappa \underline{\lambda \mu \nu}} \nabla^{\kappa} \delta A \underline{\lambda \mu \nu} .
$$

This gives then

$$
\begin{aligned}
& \delta S_{1}=-\int_{\mathbb{R}^{4}} d^{4} x \sqrt{|g|} \frac{1}{8}\left[\frac{d K(F)}{d F} \frac{1}{-F} F_{\kappa \underline{\lambda \mu \nu}} \nabla^{\kappa} \delta A \underline{\lambda \mu \nu} \nabla^{\alpha} F^{2} \nabla_{\alpha} F^{2}+\right. \\
& \left.+4 K(F) \nabla^{\alpha}\left(-F_{\underline{\kappa \mu \mu \nu}} \nabla^{\kappa} \delta A \underline{\lambda \mu \nu}\right) \nabla_{\alpha} F^{2}\right] \\
& =-\int_{\mathbb{R}^{4}} d^{4} x \sqrt{|g|} \frac{1}{8}\left[\frac{d K(F)}{d F} \frac{1}{-F} F_{\kappa \underline{\lambda \mu \nu}} \nabla^{\kappa} \delta A \underline{\lambda \mu \nu} \nabla^{\alpha} F^{2} \nabla_{\alpha} F^{2}+\right. \\
& \left.+4 K(F) \partial^{\alpha}\left(-F_{\kappa \underline{\lambda \mu \nu}} \nabla^{\kappa} \delta A \underline{\lambda \mu \nu}\right) \nabla_{\alpha} F^{2}\right] \\
& =-\int_{\mathbb{R}^{4}} d^{4} x \sqrt{|g|} \frac{1}{8}\left[\frac{d K(F)}{d F} \frac{1}{-F} F_{\kappa \underline{\lambda \mu \nu}} \nabla^{\kappa} \delta A \underline{\lambda \mu \nu} \nabla^{\alpha} F^{2} \nabla_{\alpha} F^{2}\right]+\delta S_{12} \\
& \delta S_{12}=-\int_{\mathbb{R}^{4}} d^{4} x \sqrt{|g|} \frac{4}{8} K(F) g^{\alpha \beta}\left[-\partial_{\beta}\left(\nabla_{\kappa} \delta A_{\underline{\lambda \mu \nu}}\right) F^{\kappa \underline{\lambda \mu \nu}}-\nabla_{\kappa} \delta A_{\underline{\lambda \mu \nu}} \partial_{\beta} F^{\kappa} \underline{\lambda \mu \nu}\right] \partial_{\alpha} F^{2} .
\end{aligned}
$$

The first term of $\delta S_{12}$ must be studied in detail. Definition of covariate derivate gives

$$
\begin{aligned}
\nabla_{\alpha}\left(\nabla_{\kappa} \delta A_{\underline{\lambda \mu \nu}}\right) & =\partial_{\alpha}\left(\nabla_{\kappa} \delta A_{\underline{\lambda \mu \mu}}\right)-\Gamma_{\alpha \kappa}^{\alpha} \nabla_{\dot{\alpha}} \delta A_{\underline{\lambda \mu \nu}}-\Gamma_{\alpha \underline{\alpha}}^{\dot{\alpha}} \nabla_{\kappa} \delta A_{\dot{\alpha} \underline{\mu \nu}}+ \\
& -\Gamma_{\alpha \underline{\mu}}^{\alpha} \nabla_{\kappa} \delta A_{\underline{\lambda} \dot{\alpha} \underline{\mu}}-\Gamma_{\alpha \underline{\alpha}}^{\alpha} \nabla_{\kappa} \delta A_{\underline{\lambda \mu} \dot{\alpha}} .
\end{aligned}
$$


As we assumed that $\nabla_{\kappa} \delta A_{\underline{\lambda \mu \nu}} \neq 0$ only when $\kappa \neq \underline{\lambda}, \underline{\mu}$ and $\underline{\nu}$, so we have

$$
\nabla_{\alpha}\left(\nabla_{\kappa} \delta A_{\underline{\lambda \mu \nu}}\right)=\partial_{\alpha}\left(\nabla_{\kappa} \delta A_{\underline{\lambda \mu \mu}}\right)-\left(\Gamma \frac{\kappa}{\alpha \underline{\kappa}}+\Gamma \frac{\underline{\lambda}}{\alpha \underline{\lambda}}+\Gamma \underline{\mu} \underline{\mu} \underline{\mu}+\Gamma \underline{\underline{\nu}}\right) \nabla_{\kappa} \delta A_{\underline{\lambda \mu \nu}} .
$$

Also we have



$$
\begin{aligned}
& =\partial_{\alpha} F^{\kappa} \underline{\lambda \mu \nu}+\left(\Gamma_{\underline{\alpha} \underline{\kappa}}^{\kappa}+\Gamma_{\frac{\lambda}{\alpha}}^{\underline{\lambda}}+\Gamma^{\underline{\mu} \underline{\mu}}+\Gamma_{\underline{\alpha}}^{\underline{\nu}}\right) F^{\kappa} \underline{\lambda \mu \nu} .
\end{aligned}
$$

If we now contract this with $F_{\kappa \lambda \mu \nu}$, solve the four- $\Gamma$-factor term and substitute it to the equation for to the $\nabla_{\alpha}\left(\nabla_{\kappa} \delta A_{\underline{\lambda \mu \nu}}\right)$ we get finally

$$
\begin{aligned}
& \partial_{\alpha}\left(\nabla_{\kappa} \delta \underline{A_{\lambda \mu \mu}}\right) F^{\kappa} \underline{\lambda \mu \nu}=\nabla_{\alpha}\left(\nabla_{\kappa} \delta \underline{A_{\lambda \mu \nu}}\right) F^{\kappa} \underline{\lambda \mu \nu}+\nabla_{\alpha} F^{\kappa \lambda \mu \nu} \nabla_{\kappa} \delta A_{\underline{\lambda \mu \nu}}+ \\
& -\partial_{\alpha} F^{\kappa} \underline{\lambda \mu \nu} \nabla_{\kappa} \delta A_{\underline{\lambda \mu \nu}} .
\end{aligned}
$$

For $\delta S_{12}$ we then get

$$
\begin{aligned}
& -\delta S_{12}=-\int_{\mathbb{R}^{4}} d^{4} x \sqrt{|g|} \frac{4}{8} K(F) g^{\alpha \beta} \times \\
& \times\left[\nabla_{\beta}\left(\nabla_{\kappa} \delta A_{\underline{\lambda \mu \nu}}\right) F^{\kappa} \underline{\kappa \mu \nu}+\nabla_{\beta} F^{\kappa \lambda \mu \nu} \nabla_{\kappa} \delta A_{\underline{\lambda \mu \nu}}-\partial_{\beta} F^{\kappa} \underline{\lambda \mu \nu} \nabla_{\kappa} \delta A_{\underline{\lambda \mu \nu}}+\right. \\
& \left.+\nabla_{\kappa} \delta A_{\underline{\lambda \mu \nu}} \partial_{\beta} F^{\kappa \lambda \mu \nu}\right] \partial_{\alpha} F^{2} \text {. }
\end{aligned}
$$

Due to gauss at the far boundary where $\delta A$ can be set to zero we get

$$
\begin{aligned}
-\delta S_{12}=-\int_{\mathbb{R}^{4}} d^{4} x \sqrt{|g|} \frac{4}{8}[ & -\nabla_{\kappa} \delta A_{\underline{\lambda \mu \nu}} \nabla^{\alpha}\left(F^{\kappa} \underline{\lambda \mu \nu} \partial_{\alpha} F^{2} K(F)\right)+ \\
& \left.+\nabla^{\alpha} F^{\kappa} \underline{\lambda \mu \nu} \nabla_{\kappa} \delta A_{\underline{\lambda \mu \nu}} \partial_{\alpha} F^{2} K(F)\right] \\
=+ & \int_{\mathbb{R}^{4}} d^{4} x \sqrt{|g|} \frac{4}{8} F^{\kappa} \underline{\lambda \mu \nu} \nabla^{\alpha}\left(\partial_{\alpha} F^{2} K(F)\right) \nabla_{\kappa} \delta A_{\underline{\lambda \mu \nu}} .
\end{aligned}
$$


Now we study $S_{0}$ as given in equation (4.1b) and variate it with respect to $\delta A_{\underline{\lambda \mu \nu}}$.

$$
\begin{aligned}
& \delta S_{0}=-\int_{\mathbb{R}^{4}} d^{4} x \sqrt{|g|} \frac{\partial}{\partial A_{\underline{\lambda \mu \nu}}}\left(\frac{R}{16 \pi G(F)}+\epsilon(F)\right) \delta A_{\underline{\lambda \mu \nu}} \\
& =-\int_{\mathbb{R}^{4}} d^{4} x \sqrt{|g|} \frac{\partial F}{\partial A_{\underline{\lambda \mu \nu}}}\left(\frac{R}{16 \pi} \frac{d G^{-1}(F)}{d F}+\frac{d \epsilon(F)}{d F}\right) \delta A_{\underline{\lambda \mu \nu}} \\
& =-\int_{\mathbb{R}^{4}} d^{4} x \sqrt{|g|} \frac{\partial}{\partial A_{\underline{\lambda \mu \nu}}} \sqrt{\frac{-\nabla_{[\kappa} A_{\lambda \mu \nu]} \nabla^{[\kappa} A^{\lambda \mu \nu]}}{4 !}}\left(\frac{R}{16 \pi} \frac{d G^{-1}(F)}{d F}+\frac{d \epsilon(F)}{d F}\right) \delta A_{\underline{\lambda \mu \nu}} \\
& =-\int_{\mathbb{R}^{4}} d^{4} x \sqrt{|g|} \frac{1}{2} \frac{1}{F} \frac{\partial}{\partial A_{\underline{\lambda \mu \nu}}}\left(\frac{-\nabla_{[\kappa} A_{\lambda \mu \nu]} \nabla^{[\kappa} A^{\lambda \mu \nu]}}{4 !}\right)\left(\frac{R}{16 \pi} \frac{d G^{-1}(F)}{d F}+\frac{d \epsilon(F)}{d F}\right) \delta A_{\underline{\lambda \mu \nu}} \\
& =-\int_{\mathbb{R}^{4}} d^{4} x \sqrt{|g|} \frac{1}{2} \frac{1}{F}\left\{-24 \nabla_{\kappa} \delta A_{\underline{\lambda \mu \nu}} F^{\kappa} \underline{\lambda \mu \nu}-24 g_{\kappa \kappa} g^{\underline{\underline{\lambda}} \hat{\lambda}} g^{\underline{\mu} \dot{\mu}} g^{\underline{\underline{\nu}} \dot{\nu}} g^{\underline{\kappa} \hat{\kappa}} \nabla^{\kappa} \delta A_{\underline{\lambda \mu \nu}} F_{\hat{\kappa} \lambda \dot{\mu} \dot{\nu}}\right\} \times \\
& \times \frac{1}{4 !}\left(\frac{R}{16 \pi} \frac{d G^{-1}(F)}{d F}+\frac{d \epsilon(F)}{d F}\right) \\
& =-\int_{\mathbb{R}^{4}} d^{4} x \sqrt{|g|} \frac{2}{2} \cdot \frac{\nabla_{\kappa} \delta A_{\lambda \mu \nu} F^{\kappa \lambda \mu \nu}}{-F}\left(\frac{R}{16 \pi} \frac{d G^{-1}(F)}{d F}+\frac{d \epsilon(F)}{d F}\right) .
\end{aligned}
$$

The last part comes due to definition of derivative and variation. Finally we get for $\delta S$,

$$
\begin{aligned}
\delta S & =-\int_{\mathbb{R}^{4}} d^{4} x \sqrt{|g|} \frac{F^{\kappa} \underline{\lambda \mu}}{-F}\left\{\frac{R}{16 \pi} \frac{d G^{-1}(F)}{d F}+\frac{d \epsilon(F)}{d F}+\right. \\
& \left.+\frac{1}{8} \frac{d K(F)}{d F} \partial^{\alpha} F^{2} \partial_{\alpha} F^{2}-4 F \nabla^{\alpha}\left(\frac{1}{8} K(F) \partial_{\alpha} F^{2}\right)\right\} \nabla_{\kappa} \delta A_{\underline{\lambda \mu \nu}} \\
& =-\int_{\mathbb{R}^{4}} d^{4} x \sqrt{|g|} \nabla_{\kappa}\left\{\frac { F ^ { \kappa } \underline { \underline { \lambda \nu } } } { - F } \left(\frac{R}{16 \pi} \frac{d G^{-1}(F)}{d F}+\frac{d \epsilon(F)}{d F}+\right.\right. \\
& \left.\left.+\frac{1}{8} \frac{d K(F)}{d F} \partial^{\alpha} F^{2} \partial_{\alpha} F^{2}-4 F \nabla^{\alpha}\left(\frac{1}{8} K(F) \partial_{\alpha} F^{2}\right)\right) \delta A_{\underline{\lambda \mu \nu}}\right\}+ \\
& +\int_{\mathbb{R}^{4}} d^{4} x \sqrt{|g|} \nabla_{\kappa}\left\{\frac { F ^ { \kappa } \underline { \lambda \mu \nu } } { - F } \left(\frac{R}{16 \pi} \frac{d G^{-1}(F)}{d F}+\frac{d \epsilon(F)}{d F}+\right.\right. \\
& \left.\left.+\frac{1}{8} \frac{d K(F)}{d F} \partial^{\alpha} F^{2} \partial_{\alpha} F^{2}-4 F \nabla^{\alpha}\left(\frac{1}{8} K(F) \partial_{\alpha} F^{2}\right)\right)\right\} \delta A_{\underline{\lambda \mu \nu} .}
\end{aligned}
$$


The first part of the sum is zero due gauss and we can set $\delta A$ to zero at far boundary. So we have then

$$
\begin{aligned}
\delta S & =+\int_{\mathbb{R}^{4}} d^{4} x \sqrt{|g|} \nabla_{\kappa}\left\{\frac { F ^ { \kappa } \frac { \lambda \mu \nu } { - F } } { - F } \left(\frac{R}{16 \pi} \frac{d G^{-1}(F)}{d F}+\frac{d \epsilon(F)}{d F}+\right.\right. \\
& \left.\left.+\frac{1}{8} \frac{d K(F)}{d F} \partial^{\alpha} F^{2} \partial_{\alpha} F^{2}-4 F \nabla^{\alpha}\left(\frac{1}{8} K(F) \partial_{\alpha} F^{2}\right)\right)\right\} \delta A_{\underline{\lambda \mu \nu}} .
\end{aligned}
$$

As $\frac{F^{\kappa \lambda \mu \nu}}{F}=\frac{e^{\kappa \lambda \mu \nu}}{\sqrt{|g|}}$ ie. constant, g commutes with $\nabla_{\kappa}$, and $\delta S=0$, from above follows

$$
\nabla_{\kappa}\left(\frac{R}{16 \pi} \frac{d G^{-1}(F)}{d F}+\frac{d \epsilon(F)}{d F}+\frac{1}{8} \frac{d K(F)}{d F} \partial^{\alpha} F^{2} \partial_{\alpha} F^{2}-\frac{1}{2} F \nabla^{\alpha}\left(K(F) \partial_{\alpha} F^{2}\right)\right)=0
$$

To get Einstein equations we first variate $S_{1}$ given in equation (4.2) with respect to $\delta g^{\mu \nu}$.

$$
\begin{aligned}
& S_{1}+\delta S_{1}=-\int_{\mathbb{R}^{4}} d^{4} x\left\{(\sqrt{-g}+\delta \sqrt{-g}) \frac{1}{8} K(F+\delta F)\left(g^{\alpha \beta}+\delta g^{\alpha \beta}\right) \times\right. \\
& \times\left(\nabla_{\beta}\left(\nabla_{[\kappa} A_{\lambda \hat{\mu} \hat{\nu}]} \nabla^{[\kappa} A^{\lambda \hat{\mu} \hat{\nu}]}\right)+\delta\left[\nabla_{\beta}\left(\nabla_{[\kappa} A_{\lambda \hat{\mu} \hat{\nu}]} \nabla^{[\kappa} A^{\lambda \hat{\mu} \hat{\nu}]}\right)\right]\right) \times \\
& \left.\times\left(\nabla_{\alpha}\left(\nabla_{[\hat{\kappa}} A_{\dot{\lambda} \dot{\mu} \dot{\nu}]} \nabla^{[\hat{\kappa}} A^{\tilde{\lambda} \dot{\mu} \dot{\omega}]}\right)+\delta\left[\nabla_{\alpha}\left(\nabla_{[\hat{\kappa}} A_{\dot{\lambda} \mu \dot{\mu}]} \nabla^{[\hat{\kappa}} A^{\dot{\lambda} \dot{\mu} \dot{\nu}]}\right)\right]\right)\right\} \frac{1}{4 !^{2}} \delta g^{\mu \nu} .
\end{aligned}
$$

First derive $\delta \sqrt{-g}$, we have

$$
\frac{\partial \sqrt{-g}}{\partial g^{\mu \nu}}=-\frac{\partial g}{\partial g^{\mu \nu}} \frac{1}{2} \frac{1}{\sqrt{-g}}=\frac{\partial g}{\partial g^{\mu \nu}} \frac{1}{2} g^{-1} \sqrt{-g} .
$$

Jacobi relation and relation for differential inversion of a matrix combined gives

$$
\frac{\partial g}{\partial g^{\mu \nu}}=-\frac{g_{\mu \nu}}{g^{\mu \dot{\mu}}} \frac{\partial g}{\partial g_{\mu \dot{\nu}}}
$$

and finally

$$
\delta \sqrt{-g}=-\frac{1}{2} \sqrt{-g} g_{\mu \nu} \delta g^{\mu \nu} .
$$

For $K(F+\delta F)$ we get

$$
K(F+\delta F)=K(F)+\frac{d K(F)}{d F} \delta F .
$$

To find $\delta F$ we use definition (2.1c)

$$
\delta F_{\kappa \lambda \mu \nu}=\delta F \sqrt{-g} e_{\kappa \lambda \mu \nu}+F \delta \sqrt{-g} e_{\kappa \lambda \mu \nu}
$$


where first $\delta$ is when metric is constant and we should vary $A$ and second when $\delta A=0$, thus in this $\delta g^{\mu \nu}$ case the first term is zero. So we get

$$
\delta F_{\kappa \lambda \mu \nu}=-\frac{1}{2} \sqrt{-g} g_{\alpha \beta} \delta g^{\alpha \beta} F e_{\kappa \lambda \mu \nu}=-\frac{1}{2} g_{\alpha \beta} \delta g^{\alpha \beta} F_{\kappa \lambda \mu \nu}
$$

and similarly

$$
\delta F=-\frac{1}{2} g_{\alpha \beta} \delta g^{\alpha \beta} F .
$$

Now we get for $\delta K(F)$

$$
K(F+\delta F)=K(F)-\frac{1}{2} \frac{d K(F)}{d F} F g_{\alpha \beta} \delta g^{\alpha \beta} .
$$

Later we need also $\delta F^{2}$

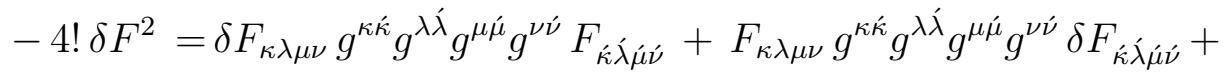

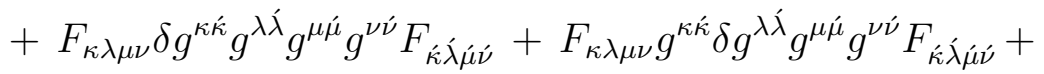

$$
\begin{aligned}
& +F_{\kappa \lambda \mu \nu} g^{\kappa \hat{\kappa}} g^{\lambda \hat{\lambda}} \delta g^{\mu \dot{\mu}} g^{\nu \dot{\nu}} F_{\kappa \dot{\kappa} \dot{\mu} \dot{\nu}}+F_{\kappa \lambda \mu \nu} g^{\kappa \hat{\kappa}} g^{\lambda \hat{\lambda}} g^{\mu \dot{\mu}} \delta g^{\nu \dot{\nu}} F_{\kappa \dot{\kappa} \dot{\mu} \dot{\nu}}= \\
& =-\frac{1}{2} g_{\alpha \beta} \delta g^{\alpha \beta} F^{2}-\frac{1}{2} g_{\alpha \beta} \delta g^{\alpha \beta} F^{2}+F_{\kappa \lambda \mu \nu} \delta g^{\kappa \kappa} F_{\kappa}{ }^{\lambda \mu \nu}+\ldots= \\
& =-g_{\alpha \beta} \delta g^{\alpha \beta} F^{2}+\frac{1}{4} g_{\kappa \kappa} \delta g^{\kappa \kappa} F^{2}+ \\
& +\frac{1}{4} g_{\dot{\lambda} \lambda} \delta g^{\lambda \dot{\lambda}} F^{2}+\frac{1}{4} g_{\dot{\mu} \mu} \delta g^{\mu \dot{\mu}} F^{2}+\frac{1}{4} g_{\dot{\nu} \nu} \delta g^{\nu \dot{\nu}} F^{2}=0 .
\end{aligned}
$$

For $\delta\left(\nabla_{\alpha} F^{2}\right)$ we get

$$
\begin{aligned}
\delta\left(\nabla_{\alpha} F^{2}\right) & =\delta\left(\nabla_{\alpha}\left(\frac{\nabla_{[\kappa} A_{\lambda \mu \nu]} \nabla^{[\kappa} A^{\lambda \mu \nu]}}{-4 !}\right)\right)=\nabla_{\alpha} \delta F^{2}+\delta \nabla_{\alpha}\left(\frac{\nabla_{[\kappa} A_{\lambda \mu \nu]} \nabla^{[\kappa} A^{\lambda \mu \nu]}}{-4 !}\right)= \\
& =\delta \nabla_{\alpha}\left(\nabla_{[\kappa} A_{\lambda \mu \nu]}\right) \frac{\nabla^{[\kappa} A^{\lambda \mu \nu]}}{-4 !}+\frac{\nabla_{[\kappa} A_{\lambda \mu \nu]}}{-4 !} \delta \nabla_{\alpha}\left(\nabla^{[\kappa} A^{\lambda \mu \nu]}\right) .
\end{aligned}
$$

as $\delta \nabla$ commutes with metric part. Using formula for $\delta \nabla_{a} \nabla_{b}$ we get

$$
\delta \nabla_{\alpha} \nabla_{[\kappa} A_{\lambda \mu \nu]}=-\frac{1}{2} F_{\kappa \lambda \mu \nu} \nabla^{c} \delta g_{\alpha c}
$$

and further

$$
\delta \nabla_{\alpha} \nabla_{[\kappa} A_{\lambda \mu \nu]} \nabla^{[\kappa} A^{\lambda \mu \nu]} \frac{1}{-4 !}=+\frac{1}{2} F^{2} \nabla^{c} \delta g_{\alpha c}
$$

Summing up all terms $(2 \mathrm{x} 4 / 4)$ we get finally for $\delta\left(\nabla F^{2}\right)$

$$
\delta\left(\nabla F^{2}\right)=-F^{2} g_{\mu \nu} \delta g^{\mu \nu} \nabla_{\alpha} .
$$


Next we gather all results together and get

$$
\begin{aligned}
\delta S_{1}= & -\int_{\mathbb{R}^{4}} d^{4} x \sqrt{-g} \frac{1}{8}\left\{-\frac{1}{2} K(F) \nabla_{\alpha} F^{2} \nabla^{\alpha} F^{2} g_{\mu \nu}+\right. \\
& +\frac{d K(F)}{d F}\left(-\frac{1}{2}\right) F \nabla_{\alpha} F^{2} \nabla^{\alpha} F^{2} g_{\mu \nu}+K(F) \nabla_{\mu} F^{2} \nabla_{\nu} F^{2}+ \\
& \left.-2 K(F) F^{2} \nabla_{\alpha} \nabla^{\alpha} F^{2} g_{\mu \nu}\right\} \delta g^{\mu \nu} .
\end{aligned}
$$

$\delta S_{0}$ can be calculated as in [10] before equation (2.3). We now combine all results (and remembering that $\delta S_{0}$ has been multiplied by a factor 2), and demand that $\delta S=0$ for any $\delta g^{\mu \nu}$. The generalized Einstein equations become

$$
\begin{aligned}
& \frac{1}{8 \pi G(F)}\left(R_{\mu \nu}-\frac{1}{2} R g_{\mu \nu}\right)+\frac{1}{16 \pi} F \frac{d G^{-1}}{d F} R g_{\mu \nu}+\frac{1}{8 \pi}\left(\nabla_{\mu} \nabla_{\nu} G^{-1}(F)-g_{\mu \nu} \square G^{-1}(F)\right)+ \\
& -\left(\epsilon(F)-F \frac{d \epsilon(F)}{d F}\right) g_{\mu \nu}-\frac{1}{8} K(F) \nabla_{\alpha} F^{2} \nabla^{\alpha} F^{2} g_{\mu \nu}-\frac{1}{8} \frac{d K(F)}{d F} F \nabla_{\alpha} F^{2} \nabla^{\alpha} F^{2} g_{\mu \nu}+ \\
& -\frac{1}{8} \cdot 2 K(F) \nabla_{\mu} F^{2} \nabla_{\nu} F^{2}-\frac{1}{8} \cdot 4 K(F) F^{2} \nabla_{\alpha} \nabla^{\alpha} F^{2} g_{\mu \nu}=0 .
\end{aligned}
$$

From Maxwell equation (4.17) we get

$$
\frac{R}{16 \pi} \frac{d G^{-1}(F)}{d F}+\frac{d \epsilon(F)}{d F}+\frac{1}{8} \frac{d K(F)}{d F} \partial^{\alpha} F^{2} \partial_{\alpha} F^{2}-\frac{1}{2} F \nabla^{\alpha}\left(K(F) \partial_{\alpha} F^{2}\right)=\mu,
$$

where $\mu$ is some constant. Now we set

$$
C(F)=F^{2} K(F) .
$$

This gives then

$$
\frac{R}{16 \pi} \frac{d G^{-1}(F)}{d F}+\frac{d \epsilon(F)}{d F}-\frac{1}{2} \frac{d C(F)}{d F} \partial^{\alpha} F \partial_{\alpha} F-C(F) \square F=\mu .
$$

For $\delta S_{1}$ we get when we substitute $C(F)$ and use again $\nabla_{\alpha} F$ instead of $\nabla_{\alpha} F^{2}$

$$
\begin{aligned}
\delta S_{1}= & -\int_{\mathbb{R}^{4}} d^{4} x \sqrt{-g} \frac{1}{8}\left\{-\frac{1}{2} C(F) \cdot 4 \cdot \nabla_{\alpha} F \nabla^{\alpha} F g_{\mu \nu}+\right. \\
& -\frac{1}{2} \frac{d C(F)}{d F} F \cdot 4 \cdot \nabla_{\alpha} F \nabla^{\alpha} F g_{\mu \nu}+\frac{1}{2} F \frac{2}{F} C(F) \cdot 4 \cdot \nabla_{\alpha} F \nabla^{\alpha} F g_{\mu \nu}+ \\
& +C(F) \cdot 4 \cdot \nabla_{\mu} F \nabla_{\nu} F-\frac{2 \cdot 2 F^{2}}{F^{2}} C(F) \nabla_{\alpha} F \nabla^{\alpha} F g_{\mu \nu}+ \\
& \left.-\frac{2 \cdot 2 F^{2}}{F^{2}} F C(F) \nabla_{\alpha} \nabla^{\alpha} F g_{\mu \nu}\right\} \delta g^{\mu \nu} .
\end{aligned}
$$


For Einstein equation (4.33) we get now

$$
\begin{aligned}
& \frac{1}{8 \pi G(F)}\left(R_{\mu \nu}-\frac{1}{2} R g_{\mu \nu}\right)+\frac{1}{16 \pi} F \frac{d G^{-1}}{d F} R g_{\mu \nu}+ \\
& +\frac{1}{8 \pi}\left(\nabla_{\mu} \nabla_{\nu} G^{-1}(F)-g_{\mu \nu} \square G^{-1}(F)\right)-\left(\epsilon(F)-F \frac{d \epsilon(F)}{d F}\right) g_{\mu \nu}+ \\
& -\frac{1}{2} C(F) g_{\mu \nu} \nabla_{\alpha} F \nabla^{\alpha} F-\frac{1}{2} F \frac{d C(F)}{d F} \nabla_{\alpha} F \nabla^{\alpha} F g_{\mu \nu}+ \\
& +C(F) \nabla_{\mu} F \nabla_{\nu} F-C(F) \square F g_{\mu \nu}=0 .
\end{aligned}
$$

From equation (4.34) we can write

$$
\frac{d \epsilon(F)}{d F}=-\frac{R}{16 \pi} \frac{d G^{-1}(F)}{d F}+\frac{1}{2} \frac{d C(F)}{d F} \nabla_{\alpha} F \nabla^{\alpha} F+C(F) \square F+\mu .
$$

Substituting the above equality we finally get a simplified form of Einstein equations

$$
\begin{aligned}
& \frac{1}{8 \pi G(F)}\left(R_{\mu \nu}-\frac{1}{2} R g_{\mu \nu}\right)+\frac{1}{8 \pi}\left(\nabla_{\mu} \nabla_{\nu} G^{-1}(F)-g_{\mu \nu} \square G^{-1}(F)\right)+ \\
& -(\epsilon(F)-\mu F) g_{\mu \nu}-\frac{1}{2} C(F) g_{\mu \nu} \nabla_{\alpha} F \nabla^{\alpha} F+C(F) \nabla_{\mu} F \nabla_{\nu} F=0 .
\end{aligned}
$$

[1] L.P. Gor'kov, On the energy spectrum of superconductors, JETP 7, 505-508 (1958).

[2] Y. Nambu and G. Jona-Lasinio, Dynamical model of elementary particles based on an analogy with superconductivity. I, Phys. Rev. 122, 345-358 (1961).

[3] V.G. Vaks, A.I. Larkin, On the application of the methods of superconductivity theory to the problem of the masses of elementary particles, JETP 13, 192-193 (1961).

[4] P.W. Higgs, Broken Symmetries and the Masses of Gauge Bosons. Phys. Rev. Lett. 13, 508-509 (1964).

[5] P.W. Anderson, Plasmons, gauge invariance, and mass. Phys. Rev. 130, 439-442 (1963).

[6] C.D. Froggatt and H.B. Nielsen, Origin of Symmetries, World Scientific, Singapore, 1991.

[7] P. Hořava, Stability of Fermi surfaces and K-theory, Phys. Rev. Lett. 95, 016405 (2005).

[8] G.E. Volovik, The Universe in a Helium Droplet, Clarendon Press, Oxford (2003).

[9] F.R. Klinkhamer and G.E. Volovik, "Self-tuning vacuum variable and cosmological constant," Phys. Rev. D 77, 085015 (2008),

[10] F.R. Klinkhamer and G.E. Volovik, "Dynamic vacuum variable and equilibrium approach in cosmology," Phys. Rev. D 78, 063528 (2008), arXiv:0806.2805.

[11] G.E. Volovik, From analogue models to gravitating vacuum, in: Analogue spacetimes: The first thirty years, eds. V. Cardoso, L.C.B. Crispino, S. Liberati, E.S. de Oliveira, M. Visser, Editoria Livraria da Fisica, Sao Paulo 2013, pp. 263-290; arXiv:1111.1155. 
[12] M.J. Duff and P. van Nieuwenhuizen, "Quantum inequivalence of different field representations," Phys. Lett. B 94, 179-182 (1980).

[13] A. Aurilia, H. Nicolai, and P.K. Townsend, "Hidden constants: The theta parameter of QCD and the cosmological constant of $N=8$ supergravity," Nucl. Phys. B 176, 509-522 (1980).

[14] (a) S.W. Hawking, "The cosmological constant is probably zero," Phys. Lett. B 134, 403 (1984); (b) M.J. Duff, "The cosmological constant is possibly zero, but the proof is probably wrong," Phys. Lett. B 226, 36 (1989); (c) Z.C. Wu, "The cosmological constant is probably zero, and a proof is possibly right," Phys. Lett. B 659, 891-893 (2008), arXiv:0709.3314 [gr-qc].

[15] M.J. Duncan and L.G. Jensen, "Four-forms and the vanishing of the cosmological constant," Nucl. Phys. B 336, 100-114 (1990).

[16] R. Bousso and J. Polchinski, "Quantization of four-form fluxes and dynamical neutralization of the cosmological constant," JHEP 0006, 006 (2000), arXiv:hep-th/0004134.

[17] A. Aurilia and E. Spallucci, "Quantum fluctuations of a 'constant' gauge field," Phys. Rev. D 69, 105004 (2004), arXiv:hep-th/0402096.

[18] B.G. Sidharth, A. Das, C.R. Das, L.V. Laperashvili and H.B. Nielsen, Topological Structure of the Vacuum, Cosmological Constant and Dark Energy, Int. J. Mod. Phys. A31, no. 34, 1630051 (2016). arXiv:1605.01169.

[19] B.G. Sidharth, A. Das, C.R. Das, L.V. Laperashvili and H.B. Nielsen, Cosmological Constant and the Vacuum Stability in the Standard Model, New Advances in Physics 10, 1-39 (2016).

[20] L.V. Laperashvili, H.B. Nielsen and C.R. Das, New results at LHC confirming the vacuum stability and Multiple Point Principle, Int. J. Mod. Phys. A31, 1650029 (2016), arXiv:1601.03231v2.

[21] D.L. Bennett, H.B. Nielsen and C.D. Froggatt, Standard model parameters from the multiple point principle and anti-GUT, arXiv:hep-ph/9710407.

[22] G.E. Volovik, Coexistence of different vacua in the effective quantum field theory and multiple point principle, JETP Lett. 79, 101 (2004), Pisma ZhETF 79, 131 (2004), arXiv:hep-ph/0309144.

[23] F.R. Klinkhamer and G.E. Volovik, "Dark matter from dark energy in $q$-theory," Pis'ma ZhETF 105, issue 2 (2017), arXiv:1612.02326.

[24] F.R. Klinkhamer and G.E. Volovik, More on cold dark matter from $q$-theory, arXiv:1612.04235.

[25] S. Weinberg, "The cosmological constant problem," Rev. Mod. Phys. 61, 1-23 (1989). 